\title{
A Framework for Periodized Nutrition for Athletics
}

\author{
Trent Stellingwerff \\ Canadian Sport Institute Pacific, Athletics Canada, and University of Victoria British Columbia
}

\author{
James P. Morton \\ Liverpool John Moores University
}

Louise M. Burke

Australian Institute of Sport and Australian Catholic University

\begin{abstract}
Over the last decade, in support of training periodization, there has been an emergence around the concept of nutritional periodization. Within athletics (track and field), the science and art of periodization is a cornerstone concept with recent commentaries emphasizing the underappreciated complexity associated with predictable performance on demand. Nevertheless, with varying levels of evidence, sport and event specific sequencing of various training units and sessions (long [macrocycle; months], medium [mesocycle; weeks], and short [microcycle; days and within-day duration]) is a routine approach to training periodization. Indeed, implementation of strategic temporal nutrition interventions (macro, meso, and micro) can support and enhance training prescription and adaptation, as well as acute event specific performance. However, a general framework on how, why, and when nutritional periodization could be implemented has not yet been established. It is beyond the scope of this review to highlight every potential nutritional periodization application. Instead, this review will focus on a generalized framework, with specific examples of macro-, meso-, and microperiodization for the macronutrients of carbohydrates, and, by extension, fat. More specifically, the authors establish the evidence and rationale for situations of acute high carbohydrate availability, as well as the evidence for more chronic manipulation of carbohydrates coupled with training. The topic of periodized nutrition has made considerable gains over the last decade but is ripe for further scientific progress and field application.
\end{abstract}

Keywords: performance, track and field, nutrient timing, macro, meso, micro

The concept and underpinnings of periodization are deeply rooted in the history of athletics (track and field). Indeed, the seminal scientists and coaches who developed the principles of periodization include Dr. Hans Selye with his General Adaptation Syndrome model (Selye, 1950), followed by Matveyev, Bonderchuck, and Bompa (contributions reviewed by Issurin, 2010). Numerous approaches to periodization have emerged from this work, including classical, block, polarized, and complex models (Issurin, 2010; Kiely, 2012). Within this diversity is a central theme: the purposeful sequencing of different training units (long [macrocycle; months], medium [mesocycle; weeks], and short [microcycle; days and within-day duration]) so that athletes can attain the desired readiness to perform optimally for targeted events on demand (Stone et al., 1981). However, it is now appreciated that the chaos and complexities of the individual, exposed to various stimuli (physical, emotional, and genetic), are probably much more complicated than most periodization purists would want to admit (Kiely,

Stellingwerff is with Canadian Sport Institute Pacific, Victoria, British Columbia, Canada; Athletics Canada, Ottawa, Ontario, Canada; and the Dept. of Exercise Science, Physical \& Health Education, University of Victoria, Victoria, British Columbia, Canada. Morton is with the Research Institute for Sport and Exercise Sciences, Liverpool John Moores University, Liverpool, United Kingdom. Burke is with the Australian Institute of Sport, Belconnen, Australia; and the Mary MacKillop Institute for Health Research, Australian Catholic University, Melbourne, Australia. Stellingwerff (tstellingwerff@csipacific.ca) is corresponding author.
2018). Furthermore, the impact of nutrition on training adaptation and performance needs to be recognized.

The 2007 International Association of Athletics Federations Nutrition Consensus presented the first formal opportunity to provide theoretical guidelines for nutrition periodization, with suggestions of the approximate energetic and macronutrient demands of different training phases within a yearly periodized training plan (Stellingwerff et al., 2007). Recently, periodized nutrition has been defined as "the planned, purposeful, and strategic use of specific nutritional interventions to enhance the adaptations targeted by individual exercise sessions or periodic training plans, or to obtain other effects that will enhance performance in the longer term (Jeukendrup, 2017a)." Athletics might be considered the sport most suited to the application of periodized nutrition, given the diversity of the bioenergetic and biomechanical demands of the numerous different events. By extension, given the unique demands of all athletic disciplines and events, it is beyond the scope of this review to highlight every potential nutrition periodization approach. Instead, we will focus on a general framework highlighting the various considerations in the implementation of periodized nutrition. This review will then highlight an emerging nutritional periodization concept around the various approaches to carbohydrate $(\mathrm{CHO})$ and fat periodization; from a macrocycle (weeks to months; e.g., chronic low-CHO high-fat [(LCHF]) to mesocycle (several days to weekly; e.g., high dietary to upregulate CHO oxidation) and microcycle $\mathrm{CHO}$ periodization approaches 
(within day and between training session; e.g., acute CHOmanipulation).

\section{Framework for Periodization of Nutrition}

The godfather of the American fitness industry Jack Lalanne has often remarked, "Exercise is King while nutrition is Queen; put them together and you have a Kingdom!" Of course, nutrition strategies play a supportive role in enhancing acute training stimuli into optimal training adaptation. For example, optimizing protein (PRO) intake, PRO quality, and timing achieves only a minor adaptive hypertrophic response without the potent stimulus of resistance exercise. However, nutrition serves an important function given that elite athletes have 1400-1800 eating occasions per year, whereas training 300-800 times. Accordingly, Figure 1 highlights a theoretical framework that is fundamental for the prioritization and optimization of nutrition periodization that practitioners can apply to a myriad of nutrition interventions. First, the coach and the entire support staff should have a full and comprehensive understanding of the event-specific physiological, neuromuscular, structural, and psychological determinants for success. Second, the performance gaps of the individual athlete should be quantified as best as possible against these performance determinants. From this construct, the coach will strategically develop the various macro(months to years), meso- (weeks to months), and microcycles (days to within days) aspects of training periodization and its specific sessions, using these as to bridge between the goals and the gap. Indeed, the integration of peer-reviewed evidence from training studies with the tacit knowledge of elite coaches (Nash \& Collins, 2006) produces the "Science" and "Art" of elite training prescription and periodization (Kiely, 2012). The periodized training schedule provides a framework for the sports nutrition professional to match nutritional strategies to support training outcomes. Table 1 highlights the rationale involved in implementing macro-, meso-, and microperiodized nutrition recommendations. Conversely, Figure 1 outlines the process and highlights several periodized nutrition examples, such as the macronutrient (CHO and PRO), micronutrient (iron), and ergogenic aid (creatine) examples of macro-, meso-, and microperiodization, respectively. However,

(a) What are the event/sport specific physiological, structural/neuromuscular and psychological determinants of success?

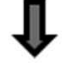

(b) What are the individual athletes gaps to these event/sport-specific success determinants?

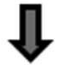

(c) What are the exercise, training and recovery periodization decisions that are long (macro; months), medium (meso; weeks), and short term (micro; days/within day) used to address the individual athletes gaps in the context of the event/sport determinants?

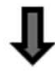

(d) What are the associated macro, meso, and micro purposefully periodized nutrition interventions that can support the periodized training and recovery stimuli or circumvent the event-specific performance determinants?

\begin{tabular}{|c|c|c|c|}
\hline $\mathrm{CHO}$ & $\begin{array}{l}\text { Macro (months) } \\
\text { - Daily CHO adjustments to match several month - } \\
\text { training phase (Inc. in general prep/ high } \\
\text { volume; Dec. in competition / low volume). }\end{array}$ & $\begin{array}{l}\text { Meso (weeks) } \\
\text { Focus of CHO based fueling during race } \\
\text { intensity endurance sessions to adapt the GI } \\
\text { over several weeks for race day fueling. }\end{array}$ & $\begin{array}{l}\text { Micro (days/within day) } \\
\text { Acute manipulation of CHO availability to } \\
\text { enhance recovery (increase) or induce } \\
\text { aerobic adaptations (decrease). }\end{array}$ \\
\hline PRO & 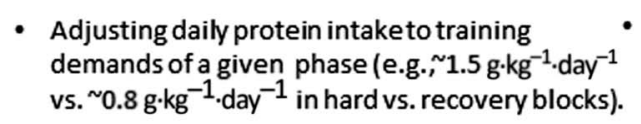 & $\begin{array}{l}\text { Increasing daily protein }\left(\sim 2{\left.\mathrm{~g} \cdot \mathrm{kg}^{-1} \cdot \mathrm{day}^{-1}\right) \text { intake}} \text { over a body composition optimization phase }\right. \\
\text { to minimize skeletal musclemass losses. }\end{array}$ & $\begin{array}{l}\text { Optimizing within day protein type, } \\
\text { amount and timing to enhance acute } \\
\text { recovery and long-term adaptation. }\end{array}$ \\
\hline Iron & $\begin{array}{l}\text { - Adequate dietary iron intake linked to the } \\
\text { stress/demands of training and individual } \\
\text { athlete iron history and profile. }\end{array}$ & $\begin{array}{l}\text { Increase in iron intake via a supplement } \\
\text { while at several week altitude camp. }\end{array}$ & $\begin{array}{l}\text { Optimizing acute iron bioavailability by } \\
\text { purposeful timing away from hard training, } \\
\text { including vitamin C, and excluding calcium. }\end{array}$ \\
\hline tine & $\begin{array}{l}\text { - Use of creatine during a 2-month hypertrophy } \\
\text { training block. }\end{array}$ & $\begin{array}{l}\text { Acute high-dose creatine loading phase to } \\
\text { enhance a several week anaerobic short } \\
\text { interval training block. }\end{array}$ & $\begin{array}{l}\text { Optimizing acute muscle creatine uptake } \\
\text { by taking posttraining with } \mathrm{CHO} \text {. }\end{array}$ \\
\hline
\end{tabular}

Figure 1 - A methodological framework required for successful nutrition periodization, including examples of macro- (months), meso- (weeks) and microcycle (days/within day) nutrition periodization interventions for carbohydrate $(\mathrm{CHO})$, protein $(\mathrm{PRO})$, iron, and creatine. Dec $=$ decreased; Inc $=$ increased; GI = gastrointestinal. 


\section{Table 1 Nutritional Considerations in Relation to Macro-, Meso- and Microcycle Training Periodization}

\author{
Performance and/or periodization considerations \\ Macroperiodization (months to weeks) \\ What is the specific load and density of this training phase/block for \\ this macrocycle (stimulus)? \\ What are the EA requirements of this macrophase? \\ In relation to the training phase/block, what are the current and long- \\ term body composition goals? Are changes even necessary? \\ Are there any macro health considerations? (injury/illness profile)
}

\section{Mesoperiodization (weeks to days)}

What is the specific load and density of this training phase/block for this mesocycle (stimulus)?

What are EA requirements of this mesophase?

If a competition block (many competitions over several days to weeks), what are the chronic to acute recovery requirements?

What environmental training interventions are being implemented in this phase?

\section{Microperiodization (days to within day)}

What is the specific load and density of this training phase/block for this microcycle (stimulus)?

What are the EA requirements of various different types of training days?

What is the typical training day schedule? (within this specific training block/phase)

What are the acute recovery requirements from a unique/specific single training session?

What are the acute recovery requirements from a single competition?

What are the training or competition specific interventions to optimize performance? (from tapering to warm-up to sport psychology)

\section{Nutritional context}

Estimation of required endogenous/exogenous substrates required during this block should guide nutrition advice. Assessment of any nutrition ergogenic aids that synergistically match the macroperiodization.

Ensure adequate EI for optimal EA. If required, assess RED-S status indicators as outlined by Mountjoy et al. (2018).

Strategic team discussions around risk and reward to optimize body composition targets, and develop an individual profile.

Target potential nutrition interventions to individual athlete health issues.

Estimation of required endogenous/exogenous substrates required due to the specific training stimuli during this block should guide nutrition advice. Consideration of any nutrition ergogenic aids that synergistically match the mesoperiodization.

Ensure adequate EI for optimal EA. If required, assess RED-S status indicators as outlined by Mountjoy et al. (2018).

During heavy competition phases, extensive logistical planning and practice is required for general, competition, and recovery nutrition interventions.

Environments (heat, cold, and altitude) dictate implementation of various periodized nutrition interventions (e.g., hydration, iron, etc.).

Assessment of required endogenous/exogenous substrates required due to the specific training stimuli during this block should guide nutrition advice. Consideration of any nutrition ergogenic aids that synergistically match the microperiodization.

Ensure adequate EI for optimal EA, appreciating that there may be day-to-day EEE and EI variability.

Understanding the individual athletes daily and weekly schedule informs micro (within hour) nutrition and supplement recommendations.

Estimation of exogenous/endogenous substrates required during individual training sessions, coupled with the training goals and phase, will inform the pre-, during, and posttraining nutrition interventions.

Generally, all recovery interventions are optimized during rounds of a competition, or throughout a competition, block to maximize subsequent performance.

Competition phase tends to offer unique nutrition periodization challenges, such as body comp optimization during tapering, optimizing recovery protocols, to acute competition specific ergogenic aids (e.g., caffeine, sodium bicarbonate, etc.).

Note. $\mathrm{EA}=\mathrm{EI}-\mathrm{EEE} /$ fat-free mass. $\mathrm{CHO}=$ carbohydrate; $\mathrm{EA}=$ energy availability; $\mathrm{EI}=$ energy intake; $\mathrm{EEE}=$ exercise energy expenditure; RED-S = Relative Energy Deficiency in Sport.

the narrative of this review will exclusively focus on energy and $\mathrm{CHO}$ periodization.

\section{Periodization of Energy Intake}

Energy intake (EI) is a primary nutritional characteristic as it (a) establishes the baseline from which intakes of the macronutrients (including muscle substrates) are derived, (b) influences the capacity of the diet to achieve micronutrient targets within nutrientdensity constraints, and (c) allows the manipulation of physique via the interaction of training and energy balance. Given that the energy cost of training is often the major determinant of an athlete's energy expenditure, and that the training load varies markedly across micro-, meso-, and macrocycles of the training plan, EI should also vary between days, weeks, and training phases. In addition, some athletes may desire to manipulate their EI in attempts to alter physical and structural characteristics (e.g., reduce body fat or body mass and/or gain muscle mass), but must appreciate the risks versus rewards of such manipulations. Accordingly, energy manipulations should be strategically integrated into the annual plan to minimize the effects on training quality or competition performance, and strategies should aim to maintain sufficient energy availability (EA) to reduce the acute and chronic issues associated with the development of Relative Energy 
Deficiency in Sport [RED-S; (Mountjoy et al., 2018)]. For a specific review of low EA and RED-S in track and field athletes, the reader is referred to review by Melin et al. (2019) in the current series.

To undertake periodization of EI, the nutrition professional and coach need to undertake a full audit of step \#3 (within Figure 1) of our framework through a nutrition lens. This assessment should include estimations of total caloric and substrate utilization for each unique type of workout, which then influences the fluctuations or manipulations of energy and macronutrient intake that needs to be integrated into daily eating patterns, as well as an appreciation of these elements over a given training meso and macrocycle. Many indirect protocols can be implemented to provide information on energy expenditure of exercise (EEE) and fuel use; these include indirect calorimetry to estimate fuel utilization linked to internal load (e.g., heart rate) and/or external load (e.g., running speed) and various technologies that can estimate caloric expenditures. It should be stressed, however, that accurate measurements of EI (Larson-Meyer et al., 2018) and the EEE and activities of daily living (Murakami et al., 2016) are extremely difficult to make; indeed, daily mismatches of as little as $300 \mathrm{kcal}(1200 \mathrm{~kJ}$; Torstveit et al., 2018), which are within the typical errors of measurement of both EI and energy expenditure, can have profound effects on EA over the long term. In light of current caution around the measurement or prescription of "optimal EA," it is both unwise and impractical to suggest that athletes should chronically "micromanage" the monitoring and manipulation of EA or EI within training or competition environments. However, having an understanding of general needs and how they fluctuate can allow the athlete to develop behavioral practices that allow EI to track with EEE (e.g., to consume recovery snacks, or within session EI, on days or during periods of higher EEE), and perhaps, to focus on energy goals during targeted periods of body composition manipulation. The emerging concept of periodization of body composition allows characteristics to be manipulated within an individualized range across different phases of the annual plan according to short- and longterm issues of training adaptation, health, and performance (Heydenreich et al., 2017; Stellingwerff, 2018). A case study of a 9-year history of body composition management of an elite female middle-distance runner (Stellingwerff, 2018) illustrates the gradual progression to optimal physique over the athlete's career as well as the manipulation within a season.

\section{Carbohydrate and Fat Periodization}

A key goal of training is to enhance the various metabolic pathways to improve the capacity and/or rate of adenosine triphosphate (ATP) production, particularly to reduce or delay the onset of factors that may make these pathways limiting for competition performance. Some of the principles by which these metabolic pathways can be enhanced include increasing the size of the storage and availability of endogenous fuels (capacity) and enhancing the body's ability to make use of exogenous fuels consumed just prior to and during exercise (power). Further metabolic enhancement can also occur by improving the delivery of nutrients and oxygen to the working muscle, reducing the accumulation of by-products that might disturb cellular homeostasis or metabolic regulation, or improving the efficiency (economy) of these pathways to produce ATP across a range of absolute and relative exercise intensities. Carefully organized dietary interventions to enhance the exercisenutrient interactions can augment the role that training alone already provides. The availability of modern laboratory technologies to investigate cellular signaling events over the past decade has expanded insights into the role of nutritional support in promoting adaptations to exercise. It is now known that many substrates, and in particular muscle glycogen and plasma free fatty acids, act not only as fuels for the exercise bout being undertaken, but also as regulators of the cellular and whole body adaptation to exercise, and specifically endurance exercise (Hansen et al., 2005; Hulston et al., 2010; Morton et al., 2009; Yeo et al., 2008). In relation to this, the reader is directed to a recent review in which a range of acute and chronically applied strategies to manipulate fat and $\mathrm{CHO}$ availability are defined and explained, at least in relation to endurance events, to address some confusion over terminology, application, and theoretical basis (Burke et al., 2018). This body of work was initially termed the "train-low (smart): compete high" paradigm and involved the integration of reduced $\mathrm{CHO}$ availability for carefully chosen training sessions to enhance markers of training adaptation, while approaching competition with high $\mathrm{CHO}$ availability to maximize performance and recovery (Burke, 2010). More recently, the concept of periodization of CHO availability has been explained using the theoretical model of "fueling for the work required" whereby $\mathrm{CHO}$ availability is adjusted in accordance with the demands and goals of the specific training session to be completed (Impey et al., 2018). With this approach, total daily $\mathrm{CHO}$ intake and its distribution over the day can be modified day-by-day and meal-by-meal (i.e., within the microcycle) to manipulate $\mathrm{CHO}$ availability for each exercise occasion.

\section{Strategies to Promote CHO Utilization During Exercise}

Carbohydrate, from muscle glycogen stores and plasma glucose, provides an effective and dominant fuel source for performance across a wide variety of events in athletics (Hawley \& Leckey, 2015). Indeed, it has been known for nearly a century that $100 \%$ CHO produces $\sim 5.5 \%$ more ATP per liter of oxygen consumed than compared with $100 \%$ fat oxidation (Krogh \& Lindhard, 1920). Therefore, the achievement of high $\mathrm{CHO}$ availability (defined as CHO stores available to provide the substrate needs of an event or training session) is a key goal of competition nutrition and the reader is directed to reviews of the individualized competition needs across events in track and field (Burke et al., 2019; Costa et al., 2019; Slater et al., 2019; Stellingwerff et al., 2019; Sygo et al., 2019). In addition to promoting training quality, undertaking sessions with high $\mathrm{CHO}$ availability can enhance the pathways of oxygen-independent glycolysis and $\mathrm{CHO}$ oxidation (Cox et al., 2010).

\section{Promoting Training Adaptation via Low $\mathrm{CHO}$ Availability}

The rationale for deliberately promoting low $\mathrm{CHO}$ availability in relation to a training session is that commencing and/or recovering from a proportion of training sessions with reduced $\mathrm{CHO}$ availability activates acute cell signaling pathways that increase many of the hallmark muscle adaptations to endurance training such as mitochondrial biogenesis, angiogenesis and increased lipid oxidation (Figure 2). The landmark studies in the field have typically manipulated preexercise muscle glycogen availability (Hansen et al., 2005; Hulston et al., 2010; Morton et al., 2009; Yeo et al., 2008), given the rationale that glycogen concentration is a potent 


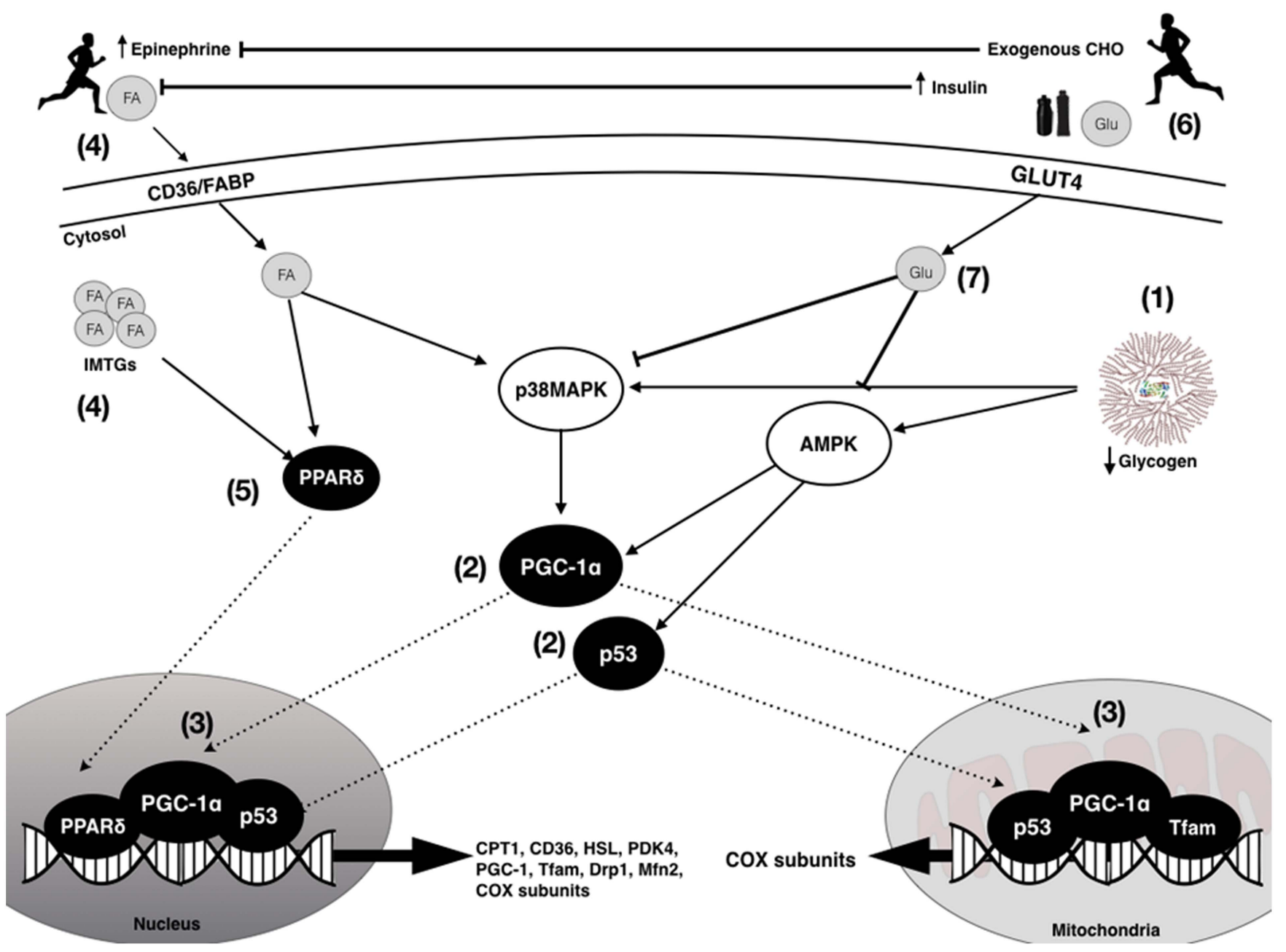

Figure 2 - Schematic overview of the potential cell signaling pathways regulating the enhanced mitochondrial adaptations associated with training with low CHO availability. (1) Reduced muscle glycogen enhances both AMPK and p38MAPK phosphorylation that results in (2) activation and translocation of PGC-1 $\alpha$ and p53 to the mitochondria and nucleus. (3) Upon entry into the nucleus, PGC-1 $\alpha$ coactivates additional transcription factors (i.e., nuclear respiratory factor $1 / 2$ ) to increase the expression of COX subunits and Tfam as well as autoregulating its own expression. In the mitochondria, PGC- $1 \alpha$ coactivates Tfam to coordinate regulation of mitochondrial DNA and induces expression of key mitochondrial proteins of the electron transport chain, for example, COX subunits. Similar to PGC-1 $\alpha$, p53 also translocates to the mitochondria to modulate Tfam activity and mitochondrial DNA expression and to the nucleus where it functions to increase expression of proteins involved in mitochondrial fission and fusion (Drp-1 and Mfn-2) and electron transport chain protein proteins. (4) Exercising in conditions of reduced CHO availability increases adipose tissue and intramuscular lipolysis via increased circulating adrenaline concentrations. (5) The resulting elevation in FFA activates the nuclear transcription factor, PPAR $\delta$ to increase expression of proteins involved in lipid metabolism such as CPT-1, PDK4, CD36, and HSL. (6) However, consuming preexercise meals rich in CHO and/or CHO during exercise increases insulin, which can downregulate lipolysis thereby negating FFA mediated signaling. (7) In addition, CHO intake before or during exercise can also reduce both AMPK and p38MAPK activity thus having negative implications for downstream regulators. AMPK $=$ AMPactivated protein kinase $\mathrm{CHO}=$ carbohydrate $; \mathrm{COX}=$ cytochrome $\mathrm{c}$ oxidase $; \mathrm{CPT} 1=$ carnitine palmitoyltransferase $1 ;$ Drp $1=$ dynamin-related protein 1 ; $\mathrm{FA}=$ fatty acid; FFA = free fatty acid; FABP = fatty acid binding protein; Glu = glucose; HSL = hormone sensitive lipase; IMTG = intramuscular triglycerides; Mfn2 = mitofusion-2; p38MAPK = mitogen-activated protein kinase; PDK4 = pyruvate dehydrogenase kinase 4; PGC-1 $\alpha=$ peroxisome proliferator-activated receptor gamma coactivator 1-alpha; PPAR $\delta=$ peroxisome proliferator-activated receptor; Tfam $=$ mitochondrial transcription factor $\mathrm{A}$.

regular of key cell signaling kinases (e.g., AMPK, p38), transcription factors (e.g., p53, PPAR) and transcriptional coactivators, for example, PGC-1 $\alpha$ (Bartlett et al., 2013; Hearris et al., 2018; Psilander et al., 2013; Yeo et al., 2010). However, it is now recognized that practical models of $\mathrm{CHO}$ periodization must extend beyond just manipulating preexercise muscle glycogen availability. Accordingly, $\mathrm{CHO}$ availability is defined as the sum of the current individual endogenous (i.e., muscle and liver glycogen) and exogenous $\mathrm{CHO}$ (i.e., $\mathrm{CHO}$ consumed before and/or during exercise) that is available to sustain the required training or racing intensity and duration (Impey et al., 2018). According to this definition, it is possible to have insufficient $\mathrm{CHO}$ availability (even if exercise is commenced with high preexercise muscle glycogen stores) if an inadequate dose of exogenous $\mathrm{CHO}$ is consumed during prolonged exercise to sustain the desired intensity (Coyle et al., 1986). Alternatively, it is possible to commence exercise with reduced muscle glycogen, but can still be considered to have sufficient $\mathrm{CHO}$ availability if the exogenous $\mathrm{CHO}$ consumed during exercise permits the completion of the desired training intensity and duration (Widrick et al., 1993). 
Table 2 Overview of Practical Approaches to Manipulate Endogenous and Exogenous CHO Availability Within $\mathrm{CHO}$ Periodization Strategies.

\begin{tabular}{lll}
\hline Practical approach & Practical implementation/theoretical rationale & Supporting references \\
\hline $\begin{array}{l}\text { Train low (glycogen) } \\
\text { session }\end{array}$ & $\begin{array}{l}\text { - Both muscle and liver glycogen are reduced during an initial morning } \\
\text { training session. CHO intake is then withheld in recovery or suboptimal } \\
\text { intakes occur such that a second session is completed in the afternoon or }\end{array}$ & $\begin{array}{l}\text { Hulston et al. (2009); Yeo et al. (2010, Morton et al. } \\
\text { (2008) }\end{array}$
\end{tabular}

Train low (fasted) session

Recovery low/sleep low strategy

Train high (glycogen + exogenous $\mathrm{CHO}$ ) session

Amalgamation of targeted $\mathrm{CHO}$ availability training and recovery approaches early evening with reduced preexercise $\mathrm{CHO}$ availability. Depending on the timing of both sessions, the total time considered in a state of low $\mathrm{CHO}$ availability could range from 3 to $8 \mathrm{hr}$.

- Commencing exercise with low muscle glycogen stores and/or sustaining exercise intensity and/or duration to a specific level of absolute glycogen depletion is associated with the activation of key cell signaling proteins (e.g., AMPK, p38, PPAR, PGC-1 $\alpha$ ), which achieve a coordinated upregulation of the nuclear and mitochondrial genomes. Over a chronic training period, this may increase oxidative enzyme protein content/activity, upregulate whole body and intramuscular lipid metabolism, with potential improvements in exercise performance and capacity.

- Breakfast is consumed after training and no form of $\mathrm{CHO}$ is consumed during exercise resulting in significant elevated FFAs. This approach would predominantly target reduced liver glycogen (associated with fasting in the overnight period) though depending on the $\mathrm{CHO}$ intake consumed in the recovery period after the last training session, pretraining muscle glycogen may also be considered low.

- Exercise undertaken in fasted conditions leads to increased metabolic stress for the muscle, central nervous system, and/or liver gluconeogenesis and leads to upregulation of AMPK and signaling pathways that increase expression of proteins regulating substrate transport (e.g., GLUT4 and CD36, FABPm, respectively) and substrate utilization (e.g., PDK4, HK, CS, $\beta$-HAD).

- Both muscle and liver glycogen are reduced during an evening training session. CHO intake is then withheld in recovery or suboptimal intakes occur such that a second session is completed on the subsequent morning with reduced preexercise $\mathrm{CHO}$ availability. Depending on the timing of both sessions, the total time considered in a state of low $\mathrm{CHO}$ availability could range from 8 to $14 \mathrm{hr}$.

- Restricting $\mathrm{CHO}$ intake in the postexercise period, therefore, maintains postexercise muscle and liver glycogen at reduced levels as well as prolongs the duration of postexercise elevations in circulating FFA availability. The interactive effects of changes in substrate availability may sustain the postexercise upregulation of cell signaling (e.g., AMPK, p53, PGC-1 $\alpha$ ) pathways thus leading to increases in the adaptive response to the session. The sleep-low train low model has been associated with improved exercise performance in trained triathletes.

- Training sessions deliberately commenced with high muscle and liver glycogen following optimal pretraining (e.g., CHO "loading" of 6-12 g/kg body mass, pretraining meal of $1-3 \mathrm{~g} / \mathrm{kg}$ body mass) and in-training fueling (e.g., CHO consumed during exercise at a rate of 30-90 g per hour).

- In this approach, promotion of high training intensity, duration, training of the gut, and practicing in competition fueling are the goals.

- The above approaches are deliberately amalgamated over a $24-$ to $48-\mathrm{hr}$ period. In this model, an initial train high session may be completed to promote training intensity and duration followed by a second train low session that is performed $24-48 \mathrm{hr}$ later with reduced $\mathrm{CHO}$ availability. The second session may have arisen as a result of a combination of sleeplow, recover low, and a low daily absolute CHO intake.

- Using this model, CHO availability can be adjusted, before, during, and/or after each training session in an attempt to take advantage of the cellular signaling responses that may occur with $\mathrm{CHO}$ restriction at each of these time periods.
Akerstrom et al. (2006); De Bock et al. (2008); Van Proeyen et al. (2011)

Bartlett et al. (2013); Marquet et al. (2016); Pilegaard et al. (2005)

Costa et al. (2017); Cox et al. (2010)

Impey et al. (2018); Stellingwerff (2012)

\footnotetext{
Note. For a summary of the terminology and rationale of different strategies of periodized fuel support for training and competition, see Burke et al. (2018) while the principles underpinning skeletal muscle adaptations, training, and performance outcomes are detailed in the review of Impey et al. (2018). AMPK = AMP-activated protein kinase; $\mathrm{B}-\mathrm{HAD}=$ beta hydroxyacyl-Coenzyme A dehydrogenase; $\mathrm{CD} 36=$ cluster of differentiation fatty acid transporter; $\mathrm{CHO}=$ carbohydrate; $\mathrm{CS}=$ citrate synthase; FABPm $=$ muscle fatty acid binding protein; FFA = free fatty acids; GLUT4 = glucose transporter 4; HK = hexokinase; PGC = peroxisome proliferator-activated receptor gamma coactivator; PPAR $\delta$ = peroxisome proliferator-activated receptor; PDK4 = pyruvate dehydrogenase kinase 4 ; p38 = mitogen-activated protein kinases .
} 


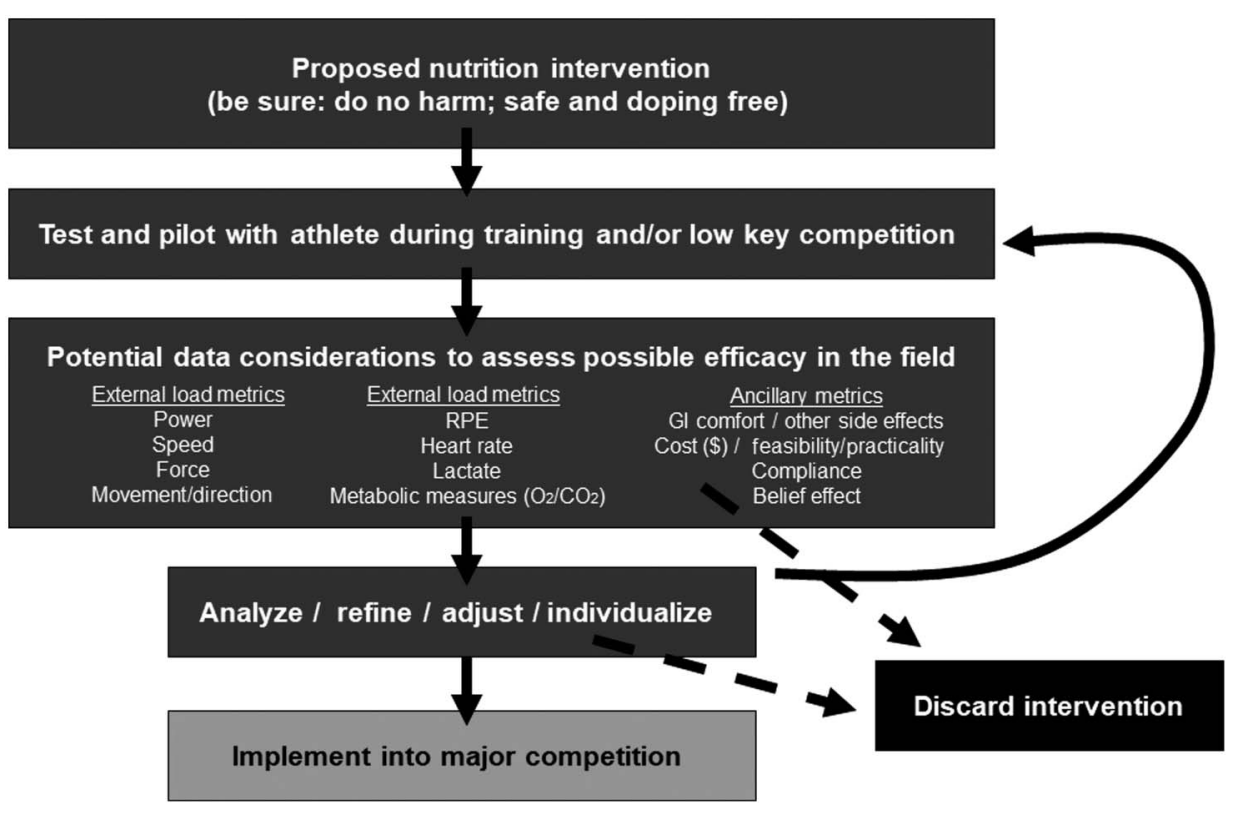

Figure 3 - Supplement individualization and piloting framework, including potential data collection variables to help inform protocol and efficacy. $\mathrm{GI}=$ gastrointestinal; RPE $=$ ratings of perceived exertion.

The various main approaches to manipulating $\mathrm{CHO}$ availability in relation to training are presented in Table 2. Essentially, it is now recognized that manipulation of both endogenous and exogenous CHO availability before (Hansen et al., 2005; Yeo et al., 2008), during (Akerstrom et al., 2006; Morton et al., 2009), and after training sessions (Marquet et al., 2016; Pilegaard et al., 2005) may lead to an augmented training response; at least in terms of acute (e.g., cell signaling) and chronic (e.g., changes in enzyme protein content and activity) skeletal muscle metabolic adaptations (Figure 2). Despite the theoretical rationale for incorporating a periodization of $\mathrm{CHO}$ availability into an elite athlete's training program, balancing the risk versus reward can be challenging for a number of reasons. First, the majority of studies to date have been conducted using cycling-based or knee extensor exercise models (78\% of 27 studies; Impey et al., 2018). These modalities may more naturally lend themselves to train-low sessions given the nonweight-bearing activity and lower eccentric loading compared with running. Second, the $\mathrm{CHO}$ requirements of the typical training sessions undertaken by elite track and field athletes are not well known and practitioners must use theoretical knowledge of glycogen utilization from relevant laboratory and field-based studies to organize appropriate manipulation of training and diet. A recent meta-analysis which provides more information in this regard clearly highlights the impact of intensity and duration on glycogen use (Areta \& Hopkins, 2018), but practitioners need to consider the use of the internal and external training load metrics outlined in Figure 3, to make best estimates. Of particular note to track and field athletes are the negative effects of reduced $\mathrm{CHO}$ (and energy) availability on markers of bone turnover (Sale et al., 2015), which contrast with the benefits of immediate postexercise feeding of CHO and PRO (Townsend et al., 2017). Although this needs to be confirmed in a longitudinal model, prolonged periods of training with reduced $\mathrm{CHO}$ availability may potentially increase the risk for stress fractures, in runners who are already at risk for RED-S and stress fractures (Heikura et al., 2018). Indeed, low CHO availability may mediate disturbed reproductive function in its own right (Loucks, 2014), creating another pathway to explain the increase in musculoskeletal injuries seen with low EA (Rauh et al., 2014). Similarly, reduced $\mathrm{CHO}$ availability during training may lead to increased susceptibility to illness owing to the role of $\mathrm{CHO}$ in modulating postexercise immune responses (Costa et al., 2005). Finally, evidence that the improved metabolic adaptations associated with the periodization of low $\mathrm{CHO}$ availability within an athlete's training program actually translates to improved performance outcomes is equivocal. Indeed, Impey et al. (2018) reported that, although periodically completing endurance-training sessions (e.g., 30-50\% of training sessions) with reduced $\mathrm{CHO}$ availability modulates the activation of acute cell signaling pathways $(73 \%$ of $n=11$ studies) and oxidative skeletal muscle adaptations (78\% of $n=9$ ), only $37 \%$ of studies demonstrate improvements in performance.

The development of practical approaches to sport-specific $\mathrm{CHO}$ periodization requires consideration of the individual athlete's training (e.g., intensity, duration, body composition, altitude, ambient temperature, etc.) and competitive goals (e.g., time required to attain and taper for peak performance) within the specific micro-, meso-, and macrocycle. An illustration of the implementation of periodized $\mathrm{CHO}$ availability in the real world was provided by a 16-week case study of three elite marathon runners (Stellingwerff, 2012). These athletes undertook a weekly average of 2.5 sessions involving low $\mathrm{CHO}$ availability during the first 12 weeks. However, during the subsequent 4-week period which focused on competition preparation, nutritional strategies shifted toward an increased frequency of practicing $\mathrm{CHO}$ fueling during training sessions (2.5 sessions per week), coupled with a reduction in low CHO training sessions (Stellingwerff, 2012). In this study, with regard to training with low $\mathrm{CHO}$ availability, fasted training was reported to be physiologically and psychologically "easier" to integrate than the more strenuous low muscle glycogen training sessions. Regardless of the specific approach, it is intuitive 
to avoid chronic periods ( $>3-5$ days), or consecutive performance, of the various "train-low" modalities due to the potential negative effects of both low $\mathrm{CHO}$ and inadequate total EA on training quality, competitive performance and health outcomes (Burke et al., 2017; Havemann et al., 2006; Mujika, 2018). Rather, careful day-to-day periodization is likely to maintain metabolic flexibility and still allow the completion of high-intensity and prolonged duration workloads on heavy training days. Intuitively, train-low may be best left to those training sessions that are not as $\mathrm{CHO}$ dependent and where the intensity and duration is not likely to be compromised by reduced $\mathrm{CHO}$ availability (e.g., steady-state type training sessions performed at intensities below the lactate threshold); however, significant work remains on how to best periodize $\mathrm{CHO}$ with the numerous training permutations (Table 2). As with all nutritional strategies, application of $\mathrm{CHO}$ periodization should only be done in conjunction with appropriately qualified nutrition professionals and be continually refined and optimized.

\section{Fat: a Relatively Unlimited Fuel Source and Driver of Adaptation}

Although endurance-trained athletes have an enhanced capacity for fat oxidation compared with untrained, this adaptation is clearly not maximized from training alone as it can be doubled, or even tripled, by chronic adaptation to a LCHF diet (Burke et al., 2002, 2017; Carey et al., 2001). Various models have been used to achieve these high fat oxidation rates including nonketogenic $\mathrm{CHO}$-restricted diet (NK-LCHF; typically 65\% energy as fat and <20\% energy from $\mathrm{CHO}$ to prevent ketosis while reducing $\mathrm{CHO}$ intake to levels below the fuel costs of daily training) and the more restrictive ketogenic LCHF diet (K-LCHF: typically $<50 \mathrm{~g} /$ day $\mathrm{CHO}$ and 75-80\% fat; Burke et al., 2018). However, studies have shown that as little as 5 days of exposure to HFLC diets, while continuing to undertake both high volume and intensity of training, achieves a robust retooling of the muscle to increase intramuscular triglycerides stores, and enhance the mobilization, transport, uptake, and oxidation of fats (for review see Burke, 2015).

By itself, however, chronic adaptation to a NK-LCHF does not translate into clear improvements in endurance performance, except in specific scenarios or individuals (Burke, 2015). Therefore, a microperiodization protocol which (theoretically) could sequentially enhance the capacity of both fat- and CHO-based fuel oxidation prior to an endurance event has been proposed; this involves a 5-6 days fat adaptation phase followed by acute restoration of $\mathrm{CHO}$ availability just prior to and during the endurance task (Burke et al., 2002; Carey et al., 2001; Havemann et al., 2006). Investigation of this protocol showed that the muscle retooling achieved by the fat-adaptation phase is robust in continuing to promote higher rates of fat oxidation in the face of aggressive strategies to restore high $\mathrm{CHO}$ availability during the endurance task (Burke et al., 2002; Carey et al., 2001). Despite substantially reduced rates of muscle glycogen utilization during the early part of the exercise task, there was no clear enhancement of a subsequent time trial in any of these studies. One apparent explanation for this outcome is that, rather than sparing glycogen utilization, chronic exposure to a high-fat diet causes an impairment of $\mathrm{CHO}$ oxidation during exercise due to a reduction on glycogenolysis and a downregulation of the activity of the pyruvate dehydrogenase complex [PDH; (Stellingwerff et al., 2006)]. Data from rodent models suggests that such inhibition of $\mathrm{PDH}$ activity may actually be due to the effects of reduced muscle glycogen availability. Indeed, commencing exercise with reduced muscle glycogen augments the activation of peroxisome proliferator-activated receptor- $\delta$ (PPAR- $\delta$; Philp et al., 2013), a transcription factor that upregulates pyruvate dehydrogenase kinase 4 (PDK4) that, in turn, phosphorylates and inactivates PDH. In human muscle, however, we have recently shown that the downregulation of $\mathrm{CHO}$ metabolism appears to be a consequence of the high-fat diet and increased fat availability rather than $\mathrm{CHO}$ restriction alone (Leckey et al., 2018). The consequences of reduced $\mathrm{CHO}$ utilization within the tricarboxylic acid cycle are likely to manifest in a reduced capacity for ATP production at high intensities. For example, in a study in which the fat adaptation-CHO restoration model was undertaken prior to a $100 \mathrm{~km}$ cycling time trial, there was a trend toward impairment of overall performance, but a significant reduction in ability to complete $1-\mathrm{km}$ and $4-\mathrm{km}$ sprints undertaken at $\sim 90 \%$ peak power output interspersed within the protocol (Havemann et al., 2006). This reduction in power output matches the $\sim 30 \%$ reduction in $\mathrm{PDH}$ and estimated glycogenolysis during a 1-min sprint at $150 \%$ of peak power output found by Stellingwerff et al. (2006).

The proposed benefits of chronic adaptation to ketogenic LCHF diets has also received recent attention in both the lay (Brukner, 2013) and the scientific press (Noakes et al., 2014; Volek et al., 2015) due to the proposed benefits of increased fat oxidation and exposure to high levels of circulating ketone bodies. Indeed, an early study featuring 4 weeks of LCHF adaptation to such $\mathrm{CHO}$ restriction showed the muscle's plasticity in being able to increase fat utilization to maintain exercise capacity at modest power outputs ( $\sim 60 \% \mathrm{VO}_{2} \mathrm{max}$; Phinney et al., 1983). However, Phinney et al. already noted that this feat was achieved in the face of impairments of capacity to undertake exercise at higher intensities. A more recent investigation of 3.5 weeks of exposure to a K-LCHF diet in elite race walkers demonstrated an apparent rationale for Phinney et al.'s findings (Burke et al., 2017). In this study, some of the highest rates for fat oxidation ever reported in the literature were achieved following adaptation to the K-LCHF diet, under conditions of either fasting or intake of fat during a prolonged exercise protocol. However, this was associated with an increase in the oxygen cost (i.e., reduced economy) of walking at a range of speeds relevant to the competitive events in this discipline (20 and $50 \mathrm{~km})$, and the K-LCHF diet did not improve 10,000 $\mathrm{m}$ performance compared with the CHO-supported dietary groups (Burke et al., 2017).

Taken together, chronic LCHF interventions have been shown to (a) decrease CHO oxidation (Burke et al., 2002; Carey et al., 2001; Havemann et al., 2006) via decreased glycogen utilization and PDH activation (Stellingwerff et al., 2006), (b) result in decreased exercise economy due to lower ATP production associated with increased fat oxidation (Burke et al., 2017), and (c) probably cause a decrease of the intestinal glucose transport proteins (SGLT-1) reducing the capacity for intestinal absorption. This would decrease the effectiveness of CHO feeding strategies, thus increasing the risk of gut disturbances (Jeukendrup, 2017b). It is important that coaches and athletes understand the metabolic demands and limiting factors in their events (Figure 1), and trial-specific interventions on an individual level (Figure 3), and realize that all sustained majority of track and field events are exceptionally $\mathrm{CHO}$ dependent. Indeed, there is continued interest in increasing rather than decreasing $\mathrm{CHO}$ dependence to improve performance of events of sustained high-intensity exercise at intensities around the "lactate threshold" (e.g., the marathon) to take advantage of the greater economy of ATP production from the oxidation of this fuel (Burke et al., 2019). 


\section{Conclusions and Future Directions}

The numerous various events in athletics, all with unique bioenergetics, biomechanical, and structural performance determinants, lend themselves to endless permutations of potential periodized nutrition interventions. Therefore, this review took a holistic approach to develop a nutrition periodization framework to guide practitioners in the field across these nutrition periodization options, while grounding them in targeted intervention first principles (Figure 1 and Table 1). From this framework, the literature has been examined regarding macro-, meso-, and microperiodization of $\mathrm{CHO}$ availability, and consequently, fat periodization (Table 2 and Figure 2), with considerations and recommendations to individualize and test in the field, as required (Figure 3). Obviously, this paper just focused on an example of nutrition periodization by examining the research in the macro-, meso-, and/or micromanipulating of $\mathrm{CHO}$, and by extension. However, Figure 1 highlights various other nutrition examples of periodization that are beyond the scope of this review, but we would highlight a few other papers in this series that also feature periodized nutrition examples for protein (Witard et al., 2019), supplements (Peeling et al., 2019), CHO fueling (Burke et al., 2019), and middle-distance athletes (Stellingwerff et al., 2018). With an appreciation of the complexities of training periodization, future directions in this field should include better quantification of knowledge and application of existing periodized approaches in elite athletes, as well as systematically controlled $\mathrm{CHO}$ periodization approaches over prolonged training blocks in larger cohorts of athletes. Other scientific areas for future investigation also include the meso and/or macroperiodization of ergogenic aids in attempts to enhance training adaptations and performance. In summary, the field of periodized nutrition has made considerable gains over the last decade, but is ripe for further progress.

\section{References}

Akerstrom, T.C., Birk, J.B., Klein, D.K., Erikstrup, C., Plomgaard, P., Pedersen, B.K., \& Wojtaszewski, J. (2006). Oral glucose ingestion attenuates exercise-induced activation of 5'-AMP-activated protein kinase in human skeletal muscle. Biochemical and Biophysical Research Communications, 342(3), 949-955. PubMed ID: 16598851 doi:10.1016/j.bbrc.2006.02.057

Areta, J.L., \& Hopkins, W.G. (2018). Skeletal muscle glycogen content at rest and during endurance exercise in humans: A meta-analysis. Sports Medicine. Advance online publication. PubMed ID: 29923148 doi:10.1007/s40279-018-0941-1

Bartlett, J.D., Louhelainen, J., Iqbal, Z., Cochran, A.J., Gibala, M.J., Gregson, W., ... Morton, J.P. (2013). Reduced carbohydrate availability enhances exercise-induced p53 signaling in human skeletal muscle: Implications for mitochondrial biogenesis. American Journal of Physiology. Regulatory, Integrative and Comparative Physiology, 304(6), R450-R458. PubMed ID: 23364526 doi:10.1152/ajpregu. 00498.2012

Brukner, P. (2013). Can elite athletes eat LCHF and win? Retrieved from https://www.youtube.com/watch?v=JMuD4Z-Oxys

Burke, L.M. (2010). Fueling strategies to optimize performance: Training high or training low? Scandinavian Journal of Medicine \& Science in Sports, 20(Suppl. 2), 48-58. doi:10.1111/j.1600-0838.2010. 01185.x

Burke, L.M. (2015). Re-Examining high-fat diets for sports performance: Did we call the 'nail in the coffin' too soon? Sports Medicine,
45(Suppl. 1), S33-S49. PubMed ID: 26553488 doi:10.1007/ s40279-015-0393-9

Burke, L.M., Hawley, J.A., Angus, D.J., Cox, G.R., Clark, S.A., Cummings, N.K., .. Hargreaves, M. (2002). Adaptations to short-term high-fat diet persist during exercise despite high carbohydrate availability. Medicine \& Science in Sports \& Exercise, 34(1), 83-91. PubMed ID: 11782652 doi:10.1097/00005768-200201000-00014

Burke, L.M., Hawley, J.A., Jeukendrup, A.E., Morton, J.P., Stellingwerff, T., \& Maughan, R.J. (2018). Commentary: Towards a universal understanding of diet-exercise strategies to manipulate fuel availability for training support and competition preparation in endurance sport. International Journal of Sport Nutrition and Exercise Metabolism, 28(5), 451-463. PubMed ID: 30249148 doi:10.1123/ijsnem.2018-0289

Burke, L.M., Jeukendrup, A.E., Jones, A.M., Bosch, A., \& Mosses, M. (2019). Contemporary nutrition strategies to optimize performance in distance runners and race walkers. International Journal of Sport Nutrition and Exercise Metabolism 29. doi:10.1123/ijsnem.2019-0004

Burke, L.M., Ross, M.L., Garvican-Lewis, L.A., Welvaert, M., Heikura, I.A., Forbes, S.G., . . Hawley, J.A. (2017). Low carbohydrate, high fat diet impairs exercise economy and negates the performance benefit from intensified training in elite race walkers. The Journal of Physiology, 595(9), 2785-2807. PubMed ID: 28012184 doi:10. 1113/JP273230

Carey, A.L., Staudacher, H.M., Cummings, N.K., Stepto, N.K., Nikolopoulos, V., Burke, L.M., \& Hawley, J.A. (2001). Effects of fat adaptation and carbohydrate restoration on prolonged endurance exercise. Journal of Applied Physiology, 91(1), 115-122. PubMed ID: 11408421 doi:10.1152/jappl.2001.91.1.115

Costa, R.J., Jones, G.E., Lamb, K.L., Coleman, R., \& Williams, J.H. (2005). The effects of a high carbohydrate diet on cortisol and salivary immunoglobulin A (s-IgA) during a period of increase exercise workload amongst Olympic and Ironman triathletes. International Journal of Sports Medicine, 26(10), 880-885. PubMed ID: 16320174 doi:10.1055/s-2005-837467

Costa, R.J., Knechtle, B., Tarnopolsky, M., \& Hoffman, M.D. (2019). Nutrition for ultramarathon running: Trail, track, and road. International Journal of Sport Nutrition and Exercise Metabolism, 29. doi:10.1123/ijsnem.2018-0255

Costa, R.J.S., Miall, A., Khoo, A., Rauch, C., Snipe, R., Camoes-Costa, V., \& Gibson, P. (2017). Gut-training: The impact of two weeks repetitive gut-challenge during exercise on gastrointestinal status, glucose availability, fuel kinetics, and running performance. Applied Physiology, Nutrition, and Metabolism, 42(5), 547-557. PubMed ID: 28177715 doi:10.1139/apnm-2016-0453

Cox, G.R., Clark, S.A., Cox, A.J., Halson, S.L., Hargreaves, M., Hawley, J.A., .. Burke, L.M. (2010). Daily training with high carbohydrate availability increases exogenous carbohydrate oxidation during endurance cycling. Journal of Applied Physiology, 109(1), 126-134. PubMed ID: 20466803 doi:10.1152/japplphysiol. 00950.2009

Coyle, E.F., Coggan, A.R., Hemmert, M.K., \& Ivy, J.L. (1986). Muscle glycogen utilization during prolonged strenuous exercise when fed carbohydrate. Journal of Applied Physiology, 61(1), 165-172. PubMed ID: 3525502 doi:10.1152/jappl.1986.61.1.165

De Bock, K., Derave, W., Eijnde, B.O., Hesselink, M.K., Koninckx, E., Rose, A.J., ... Hespel, P. (2008). Effect of training in the fasted state on metabolic responses during exercise with carbohydrate intake. Journal of Applied Physiology, 104(4), 1045-1055. PubMed ID: 18276898 doi:10.1152/japplphysiol.01195.2007

Hansen, A.K., Fischer, C.P., Plomgaard, P., Andersen, J.L., Saltin, B., \& Pedersen, B.K. (2005). Skeletal muscle adaptation: Training twice every second day vs. training once daily. Journal of Applied 
Physiology, 98(1), 93-99. PubMed ID: 15361516 doi:10.1152/ japplphysiol.00163.2004

Havemann, L., West, S.J., Goedecke, J.H., Macdonald, I.A., St Clair Gibson, A., Noakes, T.D., \& Lambert, E.V. (2006). Fat adaptation followed by carbohydrate loading compromises high-intensity sprint performance. Journal of Applied Physiology, 100(1), 194-202. PubMed ID: 16141377 doi:10.1152/japplphysiol.00813.2005

Hawley, J.A., \& Leckey, J.J. (2015). Carbohydrate dependence during prolonged, intense endurance exercise. Sports Medicine, 45(Suppl. 1), 5-12. doi:10.1007/s40279-015-0400-1

Hearris, M.A., Hammond, K.M., Fell, J.M., \& Morton, J.P. (2018). Regulation of muscle glycogen metabolism during exercise: Implications for endurance performance and training adaptations. Nutrients, 10(3), E298. PubMed ID: 29498691 doi:10.3390/nu10030298

Heikura, I.A., Uusitalo, A.L.T., Stellingwerff, T., Bergland, D., Mero, A.A., \& Burke, L.M. (2018). Low energy availability is difficult to assess but outcomes have large impact on bone injury rates in elite distance athletes. International Journal of Sport Nutrition and Exercise Metabolism, 28(4), 403-411. PubMed ID: 29252050 doi:10. 1123/ijsnem.2017-0313

Heydenreich, J., Kayser, B., Schutz, Y., \& Melzer, K. (2017). Total energy expenditure, energy intake, and body composition in endurance athletes across the training season: A systematic review. Sports Medicine Open, 3(1), 8. PubMed ID: 28161872 doi:10.1186/ s40798-017-0076-1

Hulston, C.J., Venables, M.C., Mann, C.H., Martin, C., Philp, A., Baar, K., \& Jeukendrup, A.E. (2010). Training with low muscle glycogen enhances fat metabolism in well-trained cyclists. Medicine \& Science in Sports \& Exercise, 42(11), 2046-2055. PubMed ID: 20351596 doi:10.1249/MSS.0b013e3181dd5070

Impey, S.G., Hearris, M.A., Hammond, K.M., Bartlett, J.D., Louis, J., Close, G.L., \& Morton, J.P. (2018). Fuel for the work required: A theoretical framework for carbohydrate periodization and the glycogen threshold hypothesis. Sports Medicine. 48(5), 10311048. PubMed ID: 29453741 doi:10.1007/s40279-018-0867-7

Issurin, V.B. (2010). New horizons for the methodology and physiology of training periodization. Sports Medicine, 40(3), 189-206. PubMed ID: 20199119 doi:10.2165/11319770-000000000-00000

Jeukendrup, A.E. (2017a). Periodized nutrition for athletes. Sports Medicine, 47(Suppl. 1), 51-63. PubMed ID: 28332115 doi:10.1007/ s40279-017-0694-2

Jeukendrup, A.E. (2017b). Training the gut for athletes. Sports Medicine, 47(Suppl. 1), 101-110. PubMed ID: 28332114 doi:10.1007/s40279017-0690-6

Kiely, J. (2012). Periodization paradigms in the 21st century: Evidence-led or tradition-driven? International Journal of Sports Physiology and Performance, 7(3), 242-250. PubMed ID: 22356774 doi:10.1123/ ijspp.7.3.242

Kiely, J. (2018). Periodization theory: Confronting an inconvenient truth. Sports Medicine, 48(4), 753-764. PubMed ID: 29189930 doi:10. 1007/s40279-017-0823-y

Krogh, A., \& Lindhard, J. (1920). The relative value of fat and carbohydrate as sources of muscular energy: With appendices on the correlation between standard metabolism and the respiratory quotient during rest and work. Biochemical Journal, 14(3-4), 290-363. PubMed ID: 16742941 doi:10.1042/bj0140290

Larson-Meyer, D.E., Woolf, K., \& Burke, L. (2018). Assessment of nutrient status in athletes and the need for supplementation. International Journal of Sport Nutrition and Exercise Metabolism, 28(2), 139-158. PubMed ID: 29252049 doi:10.1123/ijsnem.2017-0338

Leckey, J.J., Hoffman, N.J., Parr, E.B., Devlin, B.L., Trewin, A.J., Stepto, N.K., . . Hawley, J.A. (2018). High dietary fat intake increases fat oxidation and reduces skeletal muscle mitochondrial respiration in trained humans. The FASEB Journal, 32(6), 2979-2991. PubMed ID: 29401600 doi:10.1096/fj.201700993R

Loucks, A.B. (2014). Energy balance and energy availability. In R.J. Maughan (Ed.), Encyclopedia of sports medicine: Sports nutrition. (pp. 72-87). Oxford, UK: Wiley Blackwell.

Marquet, L.A., Brisswalter, J., Louis, J., Tiollier, E., Burke, L.M., Hawley, J.A., \& Hausswirth, C. (2016). Enhanced endurance performance by periodization of carbohydrate intake: "Sleep Low" strategy. Medicine \& Science in Sports \& Exercise, 48(4), 663-672. PubMed ID: 26741119 doi:10.1249/MSS.0000000000000823

Melin, A., Heikura, I., Tendenforte, A., \& Mountjoy, M. (2019). Energy availability in athletics: Health, performance, and physique. International Journal of Sport Nutrition and Exercise Metabolism, 29. doi:10.1123/ijsnem.2018-0201

Morton, J.P., Croft, L., Bartlett, J.D., Maclaren, D.P., Reilly, T., Evans, L., ... Drust, B. (2009). Reduced carbohydrate availability does not modulate training-induced heat shock protein adaptations but does upregulate oxidative enzyme activity in human skeletal muscle. Journal of Applied Physiology, 106(5), 1513-1521. PubMed ID: 19265068 doi:10.1152/japplphysiol.00003.2009

Mountjoy, M., Sundgot-Borgen, J., Burke, L., Ackerman, K.E., Blauwet, C., Constantini, N., . . Budgett, R. (2018). International Olympic Committee (IOC) consensus statement on relative energy deficiency in sport (RED-S): 2018 update. International Journal of Sport Nutrition and Exercise Metabolism, 28(4), 316-331. PubMed ID: 29771168 doi:10.1123/ijsnem.2018-0136

Mujika, I. (2018). Case study: Long-term low carbohydrate, high fat diet impairs performance and subjective wellbeing in a world-class vegetarian long-distance triathlete. International Journal of Sport Nutrition and Exercise Metabolism, 13, 1-6. PubMed ID: 30160554 doi:10.1123/ijsnem.2018-0124

Murakami, H., Kawakami, R., Nakae, S., Nakata, Y., Ishikawa-Takata, K., Tanaka, S., \& Miyachi, M. (2016). Accuracy of wearable devices for estimating total energy expenditure: Comparison with metabolic chamber and doubly labeled water method. JAMA Internal Medicine. 176(5), 702-703. PubMed ID: 26999758 doi:10.1001/jamainternmed. 2016.0152

Nash, C., \& Collins, D. (2006). Tacit knowledge in expert coaching: Science or art? Quest, 58, 465-477. doi:10.1080/00336297.2006. 10491894

Noakes, T., Volek, J.S., \& Phinney, S.D. (2014). Low-carbohydrate diets for athletes: What evidence? British Journal of Sports Medicine, 48(14), 1077-1078. PubMed ID: 24861453 doi:10.1136/bjsports2014-093824

Peeling, P., Castell, L.M., Derave, W., de Hon, O., \& Burke, L.M. (2019). Sports foods and dietary supplements for optimal function and performance enhancement in track-and-field athletes. International Journal of Sport Nutrition and Exercise Metabolism,29. doi:10.1123/ ijsnem.2018-0271

Philp, A., MacKenzie, M.G., Belew, M.Y., Towler, M.C., Corstorphine, A., Papalamprou, A., ... Baar, K. (2013). Glycogen content regulates peroxisome proliferator activated receptor- partial differential (PPAR-partial differential) activity in rat skeletal muscle. PLoS ONE, 8(10), e77200. PubMed ID: 24146969 doi:10.1371/journal.pone. 0077200

Phinney, S.D., Bistrian, B.R., Evans, W.J., Gervino, E., \& Blackburn, G.L. (1983). The human metabolic response to chronic ketosis without caloric restriction: Preservation of submaximal exercise capability with reduced carbohydrate oxidation. Metabolism: Clinical and Experimental, 32(8), 769-776. PubMed ID: 6865776 doi:10.1016/ 0026-0495(83)90106-3 
Pilegaard, H., Osada, T., Andersen, L.T., Helge, J.W., Saltin, B., \& Neufer, P.D. (2005). Substrate availability and transcriptional regulation of metabolic genes in human skeletal muscle during recovery from exercise. Metabolism: Clinical and Experimental, 54(8), 1048-1055. PubMed ID: 16092055 doi:10.1016/j.metabol.2005.03.008

Psilander, N., Frank, P., Flockhart, M., \& Sahlin, K. (2013). Exercise with low glycogen increases PGC-1 $\alpha$ gene expression in human skeletal muscle. European Journal of Applied Physiology, 113(4), 951-963. PubMed ID: 23053125 doi:10.1007/s00421-012-2504-8

Rauh, M.J., Barrack, M., \& Nichols, J.F. (2014). Associations between the female athlete triad and injury among high school runners. International Journal of Sports Physical Therapy, 9(7), 948-958. PubMed ID: 25540710

Sale, C., Varley, I., Jones, T.W., James, R.M., Tang, J.C., Fraser, W.D., \& Greeves, J.P. (2015). Effect of carbohydrate feeding on the bone metabolic response to running. Journal of Applied Physiology, 119(7), 824-830. PubMed ID: 26251510 doi:10.1152/japplphysiol. 00241.2015

Selye, H. (1950). Stress and the general adaptation syndrome. British Medical Journal, 1(4667), 1383-1392. PubMed ID: 15426759 doi:10.1136/bmj.1.4667.1383

Slater, G., Sygo, J., \& Jorgensen, M. (2019). SPRINTING. . . Dietary approaches to optimize training adaptation and performance. International Journal of Sport Nutrition and Exercise Metabolism, 29. doi:10.1123/ijsnem.2018-0273

Stellingwerff, T. (2012). Case study: Nutrition and training periodization in three elite marathon runners. International Journal of Sport Nutrition and Exercise Metabolism, 22(5), 392-400. PubMed ID: 23011657 doi:10.1123/ijsnem.22.5.392

Stellingwerff, T. (2018). Case-study: Body composition periodization in an olympic-level female middle-distance runner over a 9-year career. International Journal of Sport Nutrition and Exercise Metabolism, 28(4), 428-433. PubMed ID: 29140157 doi:10.1123/ijsnem.20170312

Stellingwerff, T., Boit, M.K., \& Res, P.T. (2007). Nutritional strategies to optimize training and racing in middle-distance athletes. Journal of Sports Sciences, 25(Suppl. 1), S17-S28. PubMed ID: 18049980 doi:10.1080/02640410701607213

Stellingwerff, T., Bovim, I.M., \& Whitfield, J. (2019). Contemporary nutrition interventions to optimize performance in middle-distance runners. International Journal of Sport Nutrition and Exercise Metabolism,29. PubMed ID: 30299184. doi:10.1123/ijsnem.2018-0241

Stellingwerff, T., Spriet, L.L., Watt, M.J., Kimber, N.E., Hargreaves, M., Hawley, J.A., \& Burke, L.M. (2006). Decreased PDH activation and glycogenolysis during exercise following fat adaptation with carbohydrate restoration. American Journal of Physiology-
Endocrinology and Metabolism, 290(2), E380-E388. PubMed ID: 16188909 doi:10.1152/ajpendo.00268.2005

Stone, M.H., O'Bryant, H., \& Garhammer, J. (1981). A hypothetical model for strength training. The Journal of Sports Medicine and Physical Fitness, 21(4), 342-351. PubMed ID: 7339218

Sygo, J., Kendig Glass, A., Killer, S.C., \& Stellingwerff, T. (2019). Fueling for the field: Nutrition for jumps, throws, and combined events. International Journal of Sport Nutrition and Exercise Metabolism, 29. doi:10.1123/ijsnem.2018-0272

Torstveit, M.K., Fahrenholtz, I., Stenqvist, T.B., Sylta, O., \& Melin, A. (2018). Within-day energy deficiency and metabolic perturbation in male endurance athletes. International Journal of Sport Nutrition and Exercise Metabolism, 28(4), 419-427. doi:10.1123/ijsnem. 2017-0337

Townsend, R., Elliott-Sale, K.J., Currell, K., Tang, J., Fraser, W.D., \& Sale, C. (2017). The effect of postexercise carbohydrate and protein ingestion on bone metabolism. Medicine \& Science in Sports \& Exercise, 49(6), 1209-1218. PubMed ID: 28121797 doi:10.1249/ MSS.0000000000001211

Van Proeyen, K., De Bock, K., \& Hespel, P. (2011). Training in the fasted state facilitates re-activation of eEF2 activity during recovery from endurance exercise. European Journal of Applied Physiology, 111(7), 1297-1305. PubMed ID: 21132439 doi:10.1007/s00421010-1753-7

Volek, J.S., Noakes, T., \& Phinney, S.D. (2015). Rethinking fat as a fuel for endurance exercise. European Journal of Sport Science, 15(1):13-20. doi:10.1080/17461391.2014.959564

Widrick, J.J., Costill, D.L., Fink, W.J., Hickey, M.S., McConell, G.K., \& Tanaka, H. (1993). Carbohydrate feedings and exercise performance: Effect of initial muscle glycogen concentration. Journal of Applied Physiology, 74(6), 2998-3005. PubMed ID: 8365999 doi:10.1152/ jappl.1993.74.6.2998

Witard, O.C., Garthe, I., \& Phillips, S.M. (2019). Dietary protein for training adaptation and body composition manipulation in track-andfield athletes. International Journal of Sport Nutrition and Exercise Metabolism, 29. doi:10.1123/ijsnem.2018-0267

Yeo, W.K., McGee, S.L., Carey, A.L., Paton, C.D., Garnham, A.P., Hargreaves, M., \& Hawley, J.A. (2010). Acute signalling responses to intense endurance training commenced with low or normal muscle glycogen. Experimental Physiology, 95(2), 351-358. PubMed ID: 19854796 doi:10.1113/expphysiol.2009.049353

Yeo, W.K., Paton, C.D., Garnham, A.P., Burke, L.M., Carey, A.L., \& Hawley, J.A. (2008). Skeletal muscle adaptation and performance responses to once a day versus twice every second day endurance training regimens. Journal of Applied Physiology, 105(5), 14621470. PubMed ID: 18772325 doi:10.1152/japplphysiol.90882.2008 